JOURNAL OF THEORETICAL

AND APPLIED MECHANICS

52, 4, pp. 1071-1082, Warsaw 2014

\title{
THERMAL EFFECT ON DAMAGED STAY-CABLES
}

\author{
Libin Wang, Yong Wu \\ School of Civil Engineering, Nanjing Forestry University, Nanjing, China \\ e-mail: jhwlb@163.com; wy900516@qq.com \\ DA WANG \\ Ningbo Transportation Development Prophase Office, Zhejiang, China \\ e-mail:714309267@qq.com
}

\begin{abstract}
Cables may suffer severe damage in cable-stayed bridges, leading to cable relaxation and tension loss. Such a damage effect merges in the sag augmentation or tension tightened by the thermal effect. This paper is intended to investigate the static response of damaged cables coupled with the thermal effect. With the introduction of three damage parameters such as damage position, damage degree and damage range as well as temperature parameter, the dimensionless governing equations for cables are derived and the numerical method is employed to solve the nonlinear equations. The static behavior of damaged cables due to the thermal coupling effect is analyzed, and the way to remove the thermal effect is given. It shows that the aspect ratio is the major factor is the deflection of the mid-span and horizontal force in the cable, whereas the angle inclination is the next important parameter. Cables with the natural length close to the chord distance reveal the highest sensitivity to temperature, whereas pre-tensioned and non-pre-tensioned cables are less sensitive to the thermal effect. It further demonstrates that with more damage included, the sensitive scope of the variation coefficient of the axial force with aspect ratio and temperature changes dramatically.
\end{abstract}

Keyword: stay cables, damage, thermal effect, aspect ratio

\section{Introduction}

For cable-stayed bridges, the most critical structural component is the stay-cable system because it suspends most of the girder weight and is the primary path for transmitting live loads on the girder. Bridges can be considered safe as long as no unusual force change is detected in the cable system. The alternation of force magnitude or distribution for the whole cable system should be evidently observed if severe damage occurs on the bridge structure (Mordini et al., 2008; Hua et al., 2009; Chen et al., 2012).

The success of cable-force-based strategy mentioned above depends on two conditions. The first one is the accurate measurement of the cable force while the other one is damage by the cable itself being filtered out already. Cable forces are frequently estimated from measured natural frequencies with practical formulas developed for this purpose (Zui et al., 1996). Recently, the combined effects of sag-extensibility and bending stiffness have been fully and efficiently considered to improve the accuracy of the measurement ( $\mathrm{Ni}$ et al., 2002; Ren et al., 2005; Kim and Park 2007; Kim et al., 2007; Ceballos and Prato 2008; Kangas et al., 2012; Cho et al., 2013).

Nevertheless, like in other structures, the measurement process of the cable force from the vibration-based methodology can be significantly affected by ambient temperature and other environmental factors. Moreover, changes in modal parameters due to environmental effects can be larger than those due to structural damage (Peeters et al., 2001; Kullaa 2003; Vanlanduit et al., 2005; Xia et al., 2006; Basseville et al., 2010). Therefore, differentiating the changes and 
damage due to the environment is still a challenging task in the field of bridge engineering. Many inverse-problem-based methods are applied to deal with the output data from structural health monitoring by regression techniques to get frequency-temperature relation and filter out the thermal effect (Zhou et al., 2010; Deng et al., 2010).

As a different perspective, deterministic models (Bouaanani, 2006; Lepidi et al., 2007; Treysssede, 2009, 2010; Lepidi and Gattulli, 2012) were developed from direct thermo-mechanical formulations. Such models can furnish with a valuable mechanical interpretation of the structural behavior under thermal load, which in turn may contribute to an a priori recognition of the temperature signature on damage-sensitive structural features, and therefore, remove thermal effects from response-based structural health monitoring techniques. To study the sensitivity of linear frequencies of the cable due to temperature changes, Irvine's model has been extended to investigate the thermal effects on cable dynamics by Treysssede $(2009,2010)$. Both static and dynamic responses of inclined cables were studied in details by Lepidi and Gattulli (2012) with a continuous one-dimensional model including geometric nonlinearity. The spectral properties characterizing the free dynamics are obtained. Furthermore, two thermal effects are outlined, which are distinguished by their different origins, and geometrical and static situations. For the characterization of a damaged cable, Bouaanani (2006) examined the effect of damage location and size on the modal sensitivity of suspended cables with a numerical finite-difference approach. It is shown that there is a complex relationship between the damage and the dynamic properties of cables. Another work was conducted by Lepidi et al. (2007) to deduce a series of formulas which are consistent with the expression derived by Irvine but include damage parameters. Such an approach is easy to systematically study the static and dynamic responses of damaged cables. Moreover, it can be extended to other situations such as suspension bridges (Materazzi, 2011).

However, to the best of the authors knowledge, few reports have been found in the literature about the thermal effect on the damaged cables in cable-stayed bridges. It is well known that mass and stiffness are the two vital parts for the dynamic characteristics of the cable. The damage changes the section area, leading to cable relaxation and reduction of stiffness, a critical parameter for structural dynamics. Therefore, the study on the static thermal response of a damaged cable both on force and deflection can be the primary step for further study on dynamics of cables. In this paper, the cable damage is investigated by defining characteristic parameters such as the damage position, damage range and damage degree. Other parameters such as the aspect ratio of the cable together with the damage parameters are used to establish the equilibrium equation of the cable with the damage-thermal coupling effect. The static response of the damage cable under a temperature change is analyzed in-depth. All the work provides a beneficial reference for cable damage identification, and lays the foundation for further dynamic analysis of structures with damage.

\section{Assumptions and description of cable damage}

With the assumption of uniform environmental temperature field t0, the cable is modeled as a homogeneous elastomer with the constitutive relation following Hooke's law. We further ignore the bending stiffness and shear stiffness, and only take into account the tensile stress and the longitudinal strain which are uniformly distributed in the cable at any cross section. In the absence of external constraint and loads, the arc coordinate $\mathrm{s}$ is used to describe any point location $P(s)$ throughout the cable length ranging from $s=0$ to $s=L_{0}$. The curve $C_{0}$ is an unstressed cable configuration as shown in Fig. 1.

Structural damage normally does not occur in the whole longitudinal length of the cable. Instead, it usually appears in a certain range where the sheath cracks. Following the definition 


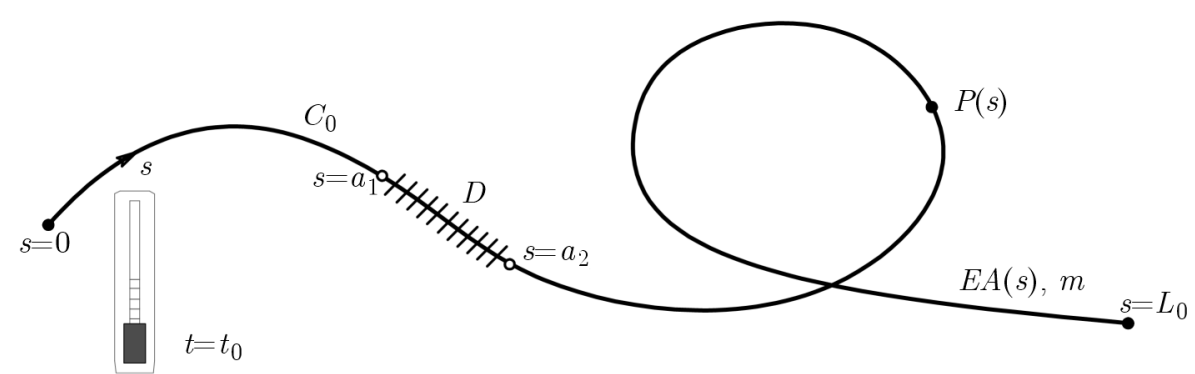

Fig. 1. Natural unstressed cable configuration $C_{0}$ and its coordinate system

on damage by Bouaanani (2006) and Lepidi et al. (2007), for a damage ranging from $a_{1}$ to $a_{2}$ along the cable $L_{0}$ as shown in Fig. 1, the damage degree, damage range and damage location are used to describe the damage.

The decrease of the cable axial stiffness $E A$ is assumed to represent the degree of cable damage. The dimensionless damage degree $\zeta(s)$ is defined as

$$
\zeta(s)=\frac{E A-E A^{d}(s)}{E A} \quad 0 \leqslant \zeta(s) \leqslant 1
$$

where $E A^{d}(s)$ is the residual axial stiffness in the cable damage area. It is understood that the existence of damage does not lead to material loss. The damage only results in a decrease in the cable axial stiffness so that the continuous cable mass and gravity density can still be used to solve the equilibrium problems.

To simplify the derivation process within any damaged region $D=\left\{s \mid a_{1}<s<a_{2}\right\}$, let us assume that the damage degree $\eta$ is uniform. Even if the existence of damage can make the mechanical models of cables complex, on this assumption, $\zeta(s)$ can be represented as a piecewise constant function

$$
\zeta(s)= \begin{cases}\eta & a_{1}<s<a_{2} \\ 0 & 0<s<a_{1} \quad \vee \quad a_{2}<s<1\end{cases}
$$

The position and the range of damage can be defined with the following formula

$$
\alpha=\frac{a_{1}+a_{2}}{2 L_{0}} \quad \delta=\frac{a_{2}-a_{1}}{L_{0}}
$$

with $0<\alpha<1$ and $0 \leqslant \delta \leqslant 1$. Thus, the parameters $\eta, \alpha$ and $\delta$ used to describe the cable damage status have been all determined.

\section{Statics equations}

Consider a cable with damage shown in Fig. 2 supported by two fixed points $A(0,0)$ and $B(L, h)$, where $L$ is the span and $h$ is the relative vertical altitude of two supports. The response of the cable under the load and temperature coupling effect allows one to obtain the new internal force and deflection of the cable. Choosing the Lagrange type arc coordinate $s$ as the only independent variable, the nature of the problem lies in establishing the function of Cartesian coordinates $x(s), z(s)$ and the cable curve arc length equation $p(s)$ as well as the axial tension equation $N(s)$ in term of $s$ on an arbitrary point $P(s)$ under the action of gravity, temperature change and damage. 


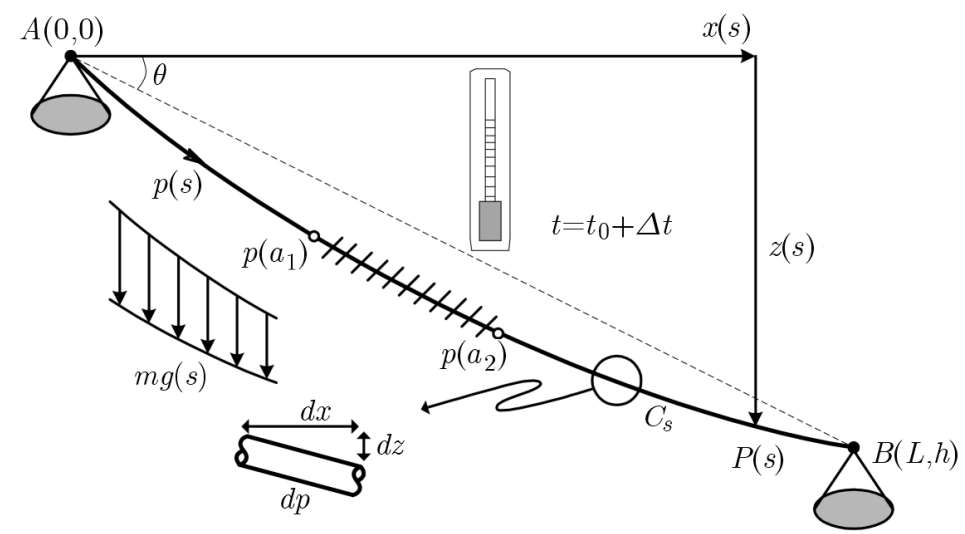

Fig. 2. The stay cable model with only one damaged segment under thermal effects

\subsection{Governing equations}

With the consideration of cable self-weight, the cable curve in the vertical plane shown in Fig. 2 must satisfy the following geometric equation

$$
\left(\frac{d x}{d p}\right)^{2}+\left(\frac{d z}{d p}\right)^{2}=1
$$

In order to meet the overall balance on the $P(s)$ left side of the cable, the following equilibrium equations are established on the horizontal and vertical directions

$$
N \frac{d x}{d p}=H \quad N \frac{d z}{d p}=V-W \frac{s}{L_{0}}
$$

where $W$ is the total weight of the cable, $N$ is the axial cable tension, $H$ is the horizontal reaction at the anchor point $A$, and $V$ is the vertical reaction, as shown in Fig. 3.

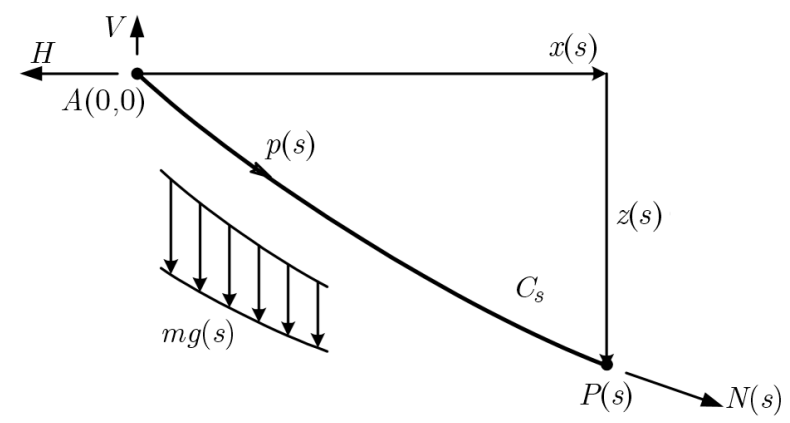

Fig. 3. The global equilibrium of the left-hand cable region with respect to the current point $P(s)$

In the elastic range, the stress-strain relation of the cable obeys Hooke's law. When the influence of temperature change and damage is introduced, the constitutive relation of the cable can be expressed as

$$
\frac{N}{E A[1-\zeta(s)]}+\beta \Delta t=\frac{d p}{d s}-1
$$

where $\beta$ is the linear expansion coefficient of the cable, and $\Delta t$ is the uniform temperature variation of the cable body caused by the environmental temperature variation.

The boundary conditions at the cable supports are

$$
x(0)=0 \quad z(0)=0 \quad \text { and } \quad x\left(L_{0}\right)=L \quad z\left(L_{0}\right)=h
$$


In addition, there are continuity conditions between the damaged and undamaged cable regions

$$
x\left(a_{i}^{-}\right)=x\left(a_{i}^{+}\right)=\lim _{\epsilon \rightarrow 0} x\left(a_{i} \pm \epsilon\right) \quad z\left(a_{i}^{-}\right)=z\left(a_{i}^{+}\right)=\lim _{\epsilon \rightarrow 0} z\left(a_{i} \pm \epsilon\right)
$$

where $\epsilon$ is a positive number less than any small amount.

\subsection{Static equation}

Combining Eqs. (3.1) and (3.2), we obtain the dimensionless axial tension in the cable

$$
n(\varsigma)=\sqrt{\hbar^{2}+(\nu-\varsigma)^{2}}
$$

by defining dimensionless parameters $n=N / W, \hbar=H / W, \nu=V / W, \varsigma=s / L_{0}$.

With the definition of $\widetilde{x}=x / L_{c}, \widetilde{z}=z / L_{c}, \rho=W / E A, \gamma=L_{0} / L_{c}, L_{c}=\sqrt{L^{2}+h^{2}}$, $\xi=(1-\eta)^{-1}, m=\beta \Delta t, \alpha_{1}=a_{1} / L_{0}, \alpha_{2}=a_{2} / L_{0}$, the dimensionless cable horizontal coordinate function can be expressed as

$$
\widetilde{x}(\varsigma)= \begin{cases}\hbar \rho \varsigma \gamma+\varphi_{1}(\varsigma) & 0 \leqslant \varsigma<\alpha_{1} \\ \hbar \rho \gamma \xi\left(\varsigma-\eta \alpha_{1}\right)+\varphi_{1}(\varsigma) & \alpha_{1} \leqslant \varsigma \leqslant \alpha_{2} \\ \hbar \rho \gamma(\varsigma+\xi \eta \delta)+\varphi_{1}(\varsigma) & \alpha_{2}<\varsigma \leqslant 1\end{cases}
$$

in which $\rho$ is the flexibility factor, and $\gamma=L_{0} / L_{c}$ is known as the cable aspect ratio. Further, the auxiliary function $\varphi_{1}(\varsigma)$ is

$$
\varphi_{1}(\varsigma)=(1+m) \hbar \gamma\left(\operatorname{arcsinh} \frac{\nu}{\hbar}-\operatorname{arcsinh} \frac{\nu-\varsigma}{\hbar}\right)
$$

Similarly, the dimensionless vertical coordinate function of the cable can be derived as

$$
\widetilde{z}(\varsigma)= \begin{cases}\rho \gamma \varsigma(\nu-\varsigma / 2)+\varphi_{2}(\varsigma) & 0 \leqslant \varsigma<\alpha_{1} \\ \rho \gamma \xi\left[\varsigma(\nu-\varsigma / 2)-\eta \alpha_{1}\left(\nu-\alpha_{1} / 2\right)\right]+\varphi_{2}(\varsigma) & \alpha_{1} \leqslant \varsigma \leqslant \alpha_{2} \\ \rho \gamma[\varsigma(\nu-\varsigma / 2)+\eta \xi \delta(\nu-\alpha)]+\varphi_{2}(\varsigma) & \alpha_{2}<\varsigma \leqslant 1\end{cases}
$$

where the auxiliary function $\varphi_{2}(\varsigma)$ reads

$$
\varphi_{2}(\varsigma)=(1+m) \hbar \gamma\left[\sqrt{1+\left(\frac{\nu}{\hbar}\right)^{2}}-\sqrt{1+\left(\frac{\nu-\varsigma}{\hbar}\right)^{2}}\right]
$$

Due to the fact that $\widetilde{x}$ and $\widetilde{z}$ are functions of two variables $\hbar$ and $\nu$ which vary as the damage state and the body temperature change, it is difficult to obtain an accurate analytical expression. The numerical method for the determination of variables $\hbar$ and $\nu$ is used herein. With the given boundary conditions, Eq. (3.5), the following equation can be deduced

$$
\begin{aligned}
& \cos \theta=\hbar \rho \gamma(1+\xi \eta \delta)+(1+m) \hbar \gamma\left(\operatorname{arcsinh} \frac{\nu}{\hbar}-\operatorname{arcsinh} \frac{\nu-1}{\hbar}\right) \\
& \sin \theta=\rho \gamma\left[\nu-\frac{1}{2}+\eta \xi \delta(\nu-\alpha)\right]+(1+m) \hbar \gamma\left[\sqrt{1+\left(\frac{\nu}{\hbar}\right)^{2}}-\sqrt{1+\left(\frac{\nu-1}{\hbar}\right)^{2}}\right]
\end{aligned}
$$

The two unknown variables $\hbar$ and $\nu$ can be numerically solved with the combination of Eqs. (3.11). Further, $\widetilde{x}(\varsigma), \widetilde{z}(\varsigma)$ and $n(\varsigma)$ can be determined correspondingly. 


\section{Parametric analysis}

All the parameters come from the existing cable-stayed bridges with the elastic modulus of the cable $E=2 \cdot 10^{5} \mathrm{MPa}$, cross sectional area $A=6000 \mathrm{~mm}^{2}$, and unit weight $\gamma_{c b}=0.08 \mathrm{MN} / \mathrm{m}^{3}$. The three damage parameters are limited in the scopes of $\eta \in[0.1,0.5]$ with its representative value of $0.2 ; \alpha \in[0.2,0.8]$ with its representative value of 0.55 ; and $\delta \in[0.05,0.25]$ with its representative value of 0.1 . The temperature variation of cable is $\Delta t \in[-30 \mathrm{~K},+30 \mathrm{~K}]$, and the corresponding thermal strain $\mathrm{m}$ lies in the range of $\left[-3.6 \cdot 10^{-4},+3.6 \cdot 10^{-4}\right]$ when the linear expansion coefficient of the cable is $\beta=1.2 \cdot 10^{-5} \mathrm{~K}^{-1}$.

\subsection{Effects of damage and temperature on the static response}

In Fig. 4, diagrams of the cable stress against temperature for different values of the aspect ratio $\gamma$ and inclined angle $\theta$ are plotted. It is shown that for a cable $\gamma=0.997$ inclined at an angle $\theta=30^{\circ}$, the axial stress is $670 \mathrm{MPa}$ at -30 degree, while for $\gamma=1.005$, the stress is only $69 \mathrm{MPa}$. Thus, the parameter $\gamma$ determines the initial stress of each cable. The larger the aspect ratio $\gamma$ is, the smaller is the initial stress.
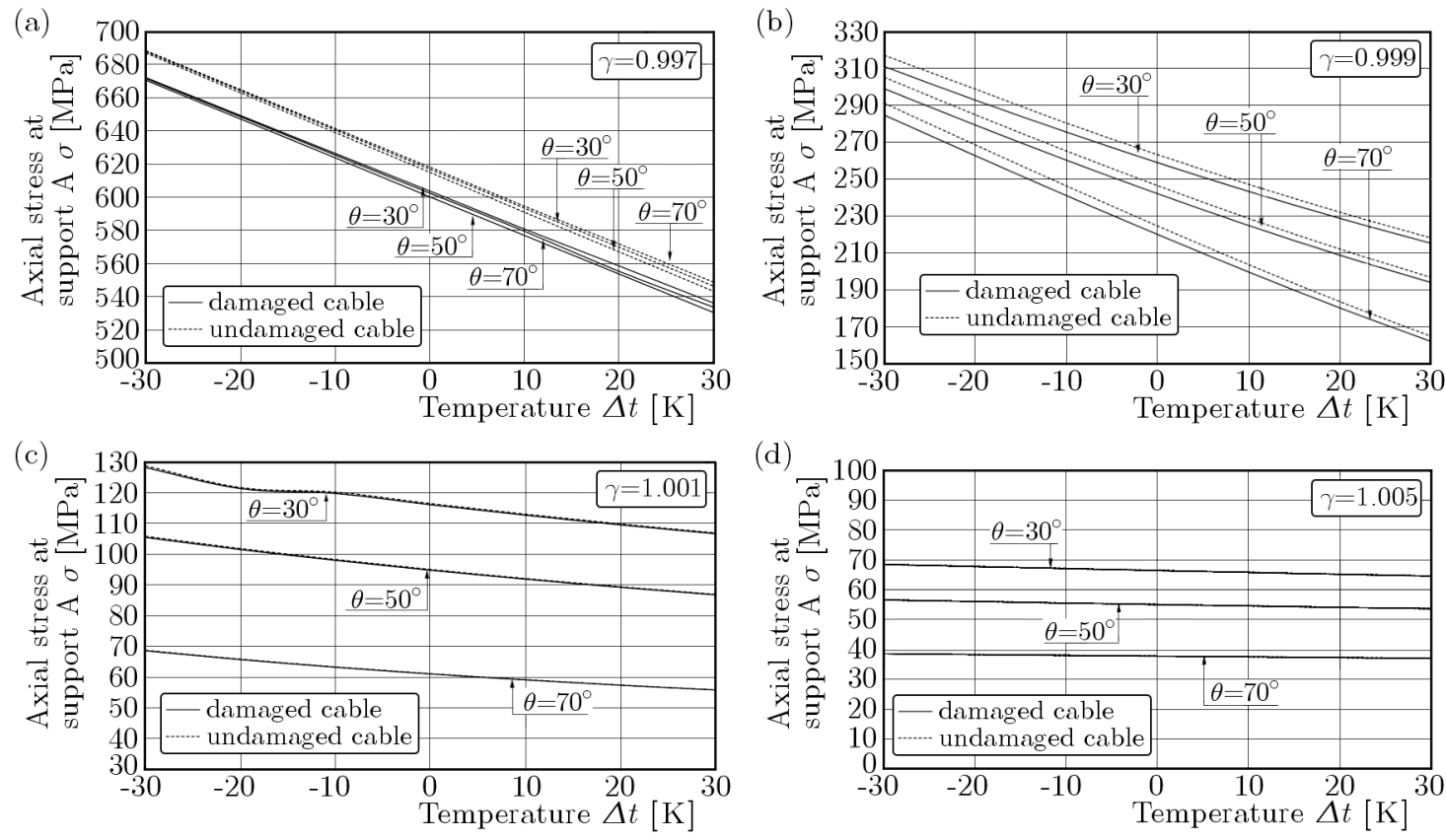

Fig. 4. Effect of temperature variation $\Delta t$, aspect ratio $\gamma$, angle and damage on cable tension

The cable stress nearly linearly decreases while $\Delta t$ increases for each cable with a different aspect ratio $\gamma$. However, there is a larger difference in the slope. For the cable with $\gamma=0.997$ and $\theta=30^{\circ}$, the slope is $-2.4 \mathrm{MPa} / \mathrm{K}$, indicating that the cable tension will drop $140 \mathrm{MPa}$ from $709 \mathrm{MPa}$ down to $560 \mathrm{MPa}$, while for the cable with $\gamma=1.005$, the slope is flat and exhibits only a $3 \mathrm{MPa}$ decrement when the temperature rises from -30 to +30 degrees as shown in Fig. 4.

The effect of damage on the cable force can also be observed in Fig. 4. For the cable with $\gamma=0.997$, the absolute decrement is by about $20 \mathrm{MPa}$, and the inclined angle has nearly no effect either on the stress of the intact or damaged cable. For cables whose $\gamma$ is larger than 0.997 but smaller than 1 , the inclined angle has an apparent effect on cable tension for both the undamaged and damaged cable as in Fig. 4b. For cables with $\gamma$ larger than 1, the angles only effect the initial stress in undamaged cables as shown in Figs. 4c and 4d. To sum up, the effect 
of damage on the cable force is different for cables with different aspect ratios $\gamma$ or in different inclined angles.

Figure 5 demonstrates the deflection due to damage and temperature for cable inclinations 0 and 70 degrees. The deflection of the cable inclined at 70 degrees is much smaller than that of the horizontal cable. The deflection due to temperature change is dramatically larger than that by damage in the representative value. The deflection increment rate is $0.020 \mathrm{~m} / \mathrm{K}$ in the horizontal cable in its mid-span, while in the cable with the angle of 70 decreases to $0.015 \mathrm{~m} / \mathrm{K}$. Thus the sag $f$ at the mid-span are the data being easily measured on-site. In the following sections, the sag in the mid-span as well as the horizontal force $\hbar_{d, t}$ at the anchor point has been investigated instead of the configuration of the entire cable.
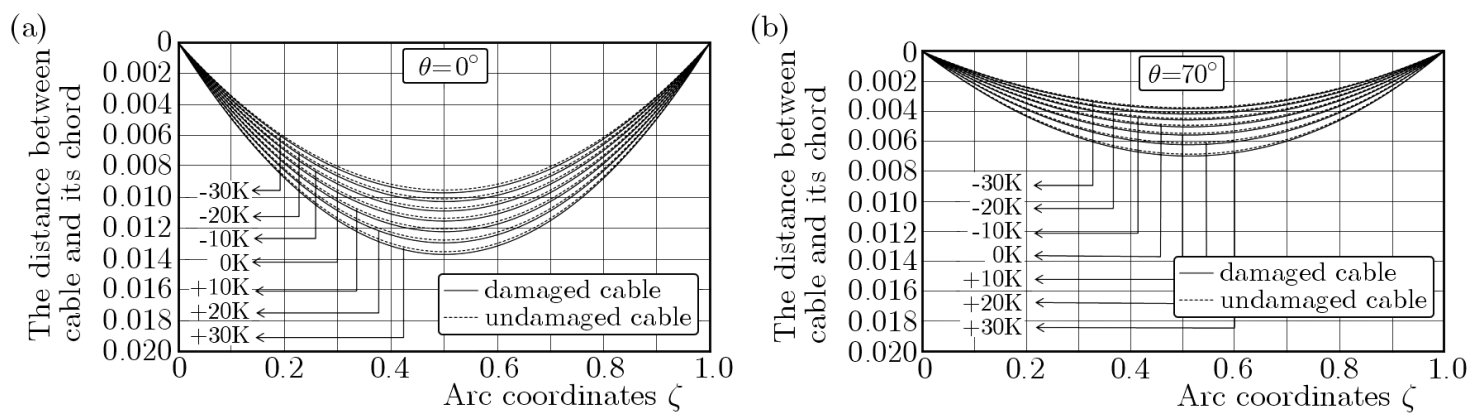

Fig. 5. Effect of damage and temperature on cable deflection

\subsection{Damage isolation form the coupling effect}

The tension $\hbar_{d, t}$ and sag of the damaged cable $f_{d, t}$ at current temperature can be expressed in terms of the corresponding values $\hbar_{u, r t}$ and $f_{u, r t}$ of the undamaged cable at the reference temperature by introducing the variation coefficient of the axial force $\chi^{2}$ and the variation coefficient of the middle sag $\kappa^{2}$

$$
\hbar_{d, t}=\chi^{2} \hbar_{u, r t} \quad f_{d, t}=\kappa^{2} f_{u, r t}
$$

The synthetic parameters $\chi^{2}$ and $\kappa^{2}$ are the ratios between the damaged cable at any current temperature to undamaged values at the reference temperature in the cable erection period, and taken as zero for convenience in this paper. Thus $\chi^{2}$ and $\kappa^{2}$ include both the thermal effect and damaged effect simultaneously. They depend not only on the damage degree $\eta$ and damage range $\delta$ but also on the cable aspect ratio $\gamma$ which is relevant to the Irvine parameter (Lepidi and Gattulli, 2012).

Equation $(4.1)_{2}$ establishes the relationship between the deflection at any temperature of a damaged cable and an intact cable at the reference erection stage temperature. As mentioned above, the deflection at the midpoint is larger enough to be easily measured on-site. When the thermal effect is filtered out, the damage defect can be detected.

\subsubsection{Thermal effect only}

The response of different cables to temperature variations can be reflected in $\chi^{2}-\gamma$ and $\kappa^{2}-\gamma$ curves as shown in Fig. 6.

It can been seen in Fig. 6a that, for the intact cable, when the aspect ratio $\gamma$ is of certain value, the temperature increases (or decreases) accordingly to $t_{0}$, and the variation coefficient of the axial force $\chi^{2}$ is less than one (or greater than one, meaning that the axial force decreases or increases). When the temperature goes up or down by the same amount from the reference temperature, the variation of the axial force (increase or decrease) is not strictly symmetric with 

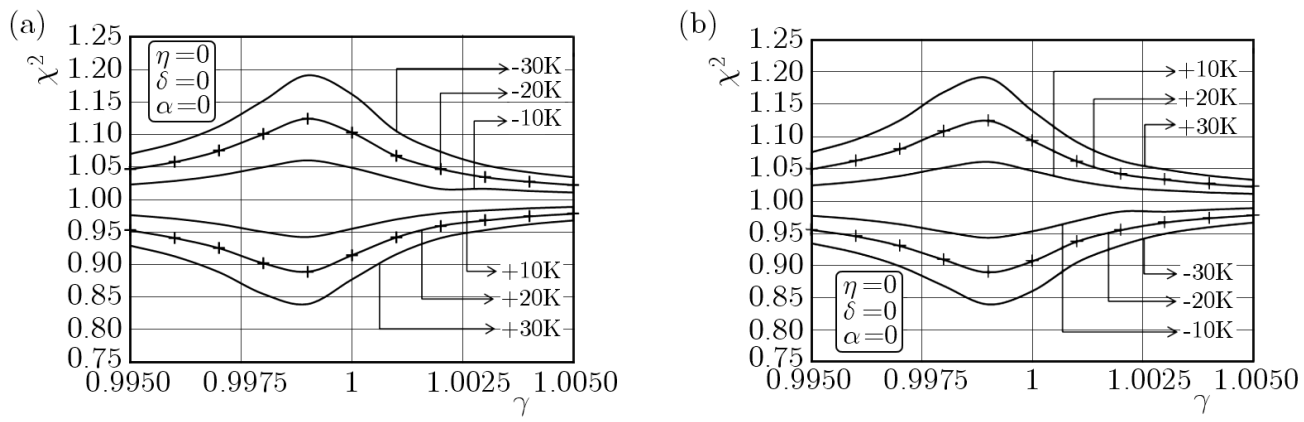

Fig. 6. Temperature effect on $\chi^{2}$ and $\kappa^{2}$ of an intact cable (pluses mark finite element results);

(a) $\chi^{2}-\gamma$ curve, (b) $\kappa^{2}-\gamma$ curve

respect to the tension value at $t=t_{0}$, and usually, the change of the axial force from cooling is greater than that from heating.

For any given temperature below zero, the curve of $\chi^{2}$ is a convex curve of $\gamma$. The peak value occurs at $\gamma=0.999$ or so, which means that $\chi^{2}$ is much more sensitive to temperature changes in the range from 0.9985 to 0.9995 . When $\gamma$ approaches down to 0.995 or increases to 1.005 , each curve trends to be parallel to each other gradually, indicating that highly pre-stressed cables or more relaxed cables are not sensitive to temperature anymore. Furthermore, the curves become more compacted when $\gamma$ equals 1.005 than when it equals 0.995 , which shows that the change of tension in relaxed cables is even smaller than in cables strongly tensioned. All features are in coincidence with examples given in Fig. 4.

Figure $6 \mathrm{~b}$ further shows the change of the sag with $\gamma$ and temperature. It is basically the same as for the undamaged curves only that when temperature goes up, the $\kappa^{2}$ is larger than one while $\chi^{2}$ is less than one. It is an interesting finding that the product of $\chi^{2}$ and $\kappa^{2}$ equals one, $\chi^{2}=\kappa^{2}=1$. To verify it, we take any curve such as the $\Delta t=+20 \mathrm{~K}$ curve in Fig. 6 as an example. All the numerical calculation results are listed in Table 1.

Table 1. The product of $\chi^{2}$ and $\kappa^{2}$

\begin{tabular}{|c|c|c|c|c|c|c|c|c|c|c|c|}
\hline$\gamma$ & 0.995 & 0.996 & 0.997 & 0.998 & 0.999 & 1.000 & 1.001 & 1.002 & 1.003 & 1.004 & 1.005 \\
\hline$\chi^{2}$ & 0.930 & 0.919 & 0.904 & 0.884 & 0.877 & 0.909 & 0.939 & 0.958 & 0.967 & 0.974 & 0.978 \\
\hline$\kappa^{2}$ & 1.075 & 1.088 & 1.106 & 1.132 & 1.141 & 1.101 & 1.065 & 1.044 & 1.034 & 1.027 & 1.023 \\
\hline$\chi^{2} \kappa^{2}$ & 1.000 & 1.000 & 1.000 & 1.001 & 1.001 & 1.001 & 1.001 & 1.000 & 1.000 & 1.000 & 1.000 \\
\hline
\end{tabular}

The reciprocal relationship between the sag and tension force provides a convenient way for field tests using the midspan sag measurement and cable force measurement at the anchor point by a pressure sensor to verify each other. It also holds true for damaged cables at any temperature.

A finite element method is used to verify the results from the theoretical method. The element is link8 in ANSYS Software and the $L_{c}$ is 200 meter long with the temperature variation $\Delta t= \pm 20 \mathrm{~K}$. The results are shown in Fig. 6 using "+". It is clear that a good fit exists between the two results.

\subsubsection{Damage effect}

In Fig. 7, the cable tension and deflection for a light damage $\eta=0.2, \delta=0.1$ and $\alpha=0.55$ at six different temperatures are plotted, while a severe damage $\eta=0.5, \delta=0.25$ and $\alpha=0.55$ is presented in Fig. 8. It is clear that for a slightly damaged cable the shape of the whole family of the curves is still much like the intact cable curves that can be seen in Figs. 7 and 6 . It is shown that both the stress and the sag generated only by the damage is much smaller than that 
due to temperature. But with the development of the damage, the shape of the curve becomes dramatically different from that of intact cables. The whole family of the curves move downwards and the peaks of the curves move to the left and right when temperature rises and decreases, respectively, showing that the damage effect becomes more dominant, and that the existence of damage not only can reduce the axial force but also can change the sensitivity of the axial force to temperature changes. Figure 8 shows the temperature effect for the cable with $\gamma=0.995$ whose stress is $1000 \mathrm{MPa}$ at the serious damage case $\eta=0.5, \delta=0.25$ and $\alpha=0.55$. Even for temperature decreasing doen to -30 degree, $\chi^{2}$ still goes down to 0.86 which should be 1.04 for the intact case. The difference 0.18 is due to damage. It is demonstrated that if we overlap Fig. 7, Fig. 8 and Fig. 6a together, the difference between the $\chi^{2}$ from each figure is just the lost tension caused by the damage. Therefore, the coupling effect is separated into the thermal effect and damage effect completely. It is useful for damage monitoring.
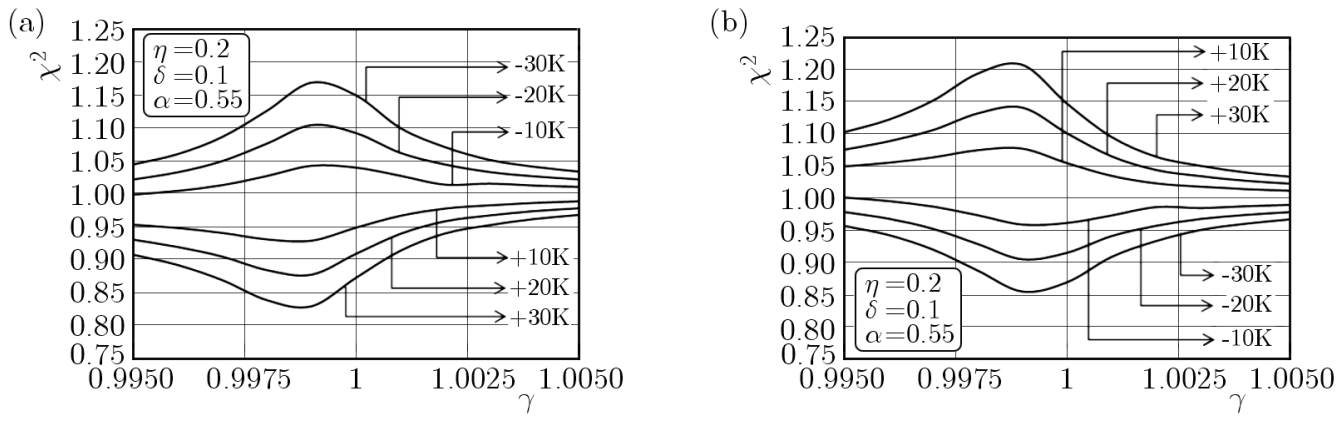

Fig. 7. Temperature effect on $\chi^{2}$ and $\kappa^{2}$ of a light damaged cable; (a) $\chi^{2}-\gamma$ curve, (b) $\kappa^{2}-\gamma$ curve

(a)

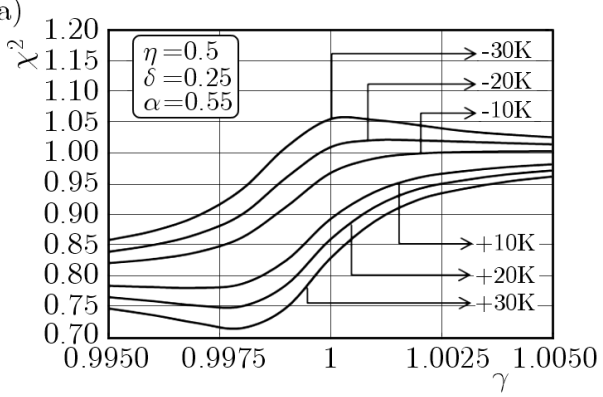

(b)

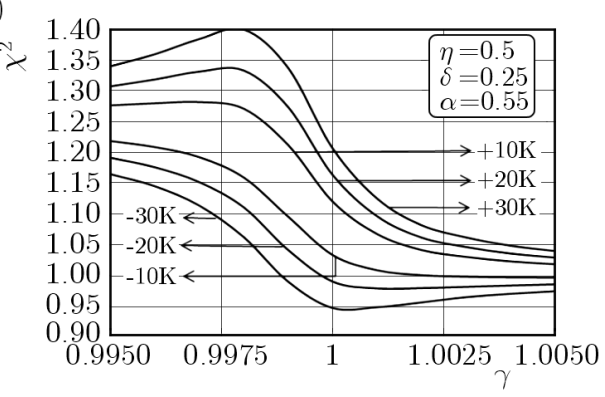

Fig. 8. Temperature effect on $\chi^{2}$ and $\kappa^{2}$ of a severely damaged cable; (a) $\chi^{2}-\gamma$ curve, (b) $\kappa^{2}-\gamma$ curve

If we neglect the thermal effect, the exact value of $\kappa^{2}$ and $\chi^{2}$ is caused only by damage. Since $\kappa^{2}$ and $\chi^{2}$ are determined by the damage degree and damage range only, using Eqs. (3.2) to (3.6) and neglecting the temperature parameters, the influence of $\eta$ and $\delta$ on $\kappa^{2}$ and $\chi^{2}$ is shown in Fig. 9.

Figure 9 presents all damage coupled to different $\kappa^{2}$ and $\chi^{2}$ values for a cable with $\gamma=0.999$. For any couple $\eta$ and $\delta$, the $\kappa^{2}$ and $\chi^{2}$ can be looked up in Fig. 9. For example, the cable force and sag are down to 0.8 and up to 1.2 of their original value, respectively, for $\eta=0.5$ and $\delta=0.25$.

It is clear for a certain $\delta$ (or $\eta$ ), the decrement rate of $\chi^{2}$ (or $\left.\kappa^{2}\right)$ is increasing with the other parameter $\eta$ (or $\delta$ ) increasing, which indicates that the response of the cable to the damage is more sensitive with damage becoming more severe. 


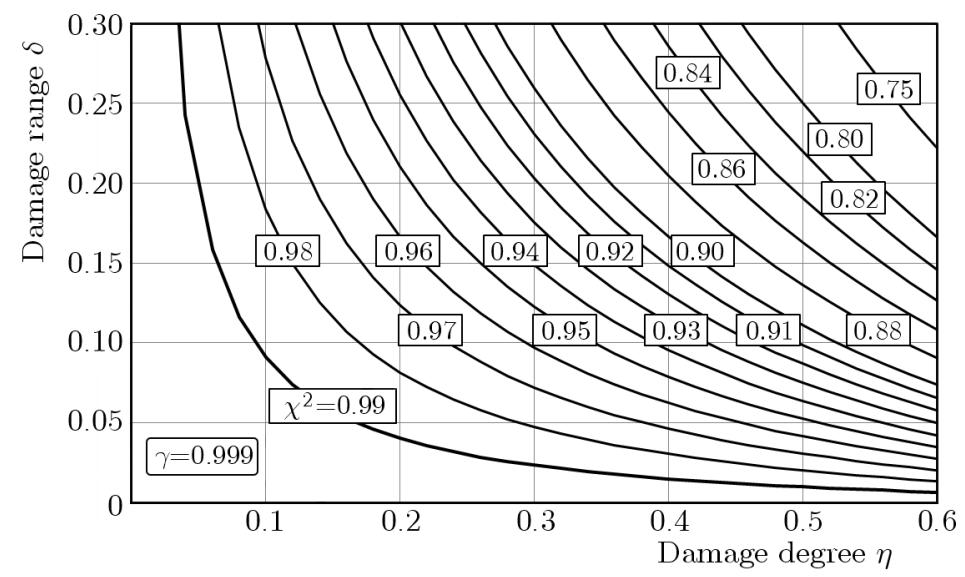

Fig. 9. $\chi^{2}$ and $\kappa^{2}$ for different couples $(\eta, \delta)$ at the given $\gamma$

\section{Conclusions}

With the damage and temperature parameters simultaneously integrated into the governing equations of the cable, the mechanical responses due to damage and the thermal coupling effect are analyzed, and the conclusions are drawn as follows.

The relationship of cable tension and deflection at the mid-span with the aspect ratio $\gamma$ at any temperature with the reference temperature is set up by two synthetic factors defined for that purpose. The study shows that cables with the natural length close to the chord distance reveal the highest sensitivity to temperature, whereas pre-tensioned and non-pre-tensioned cables are gradually less sensitive to thermal effects. The tension decrement and deflection increment due to the thermal effect are larger than those due to damage. The sensitivity of cable tension to temperature of the damaged cable is different from that of the intact cable. The damage is distinguished by decomposing the thermal and damage coupling effect. Both the thermal and damage effects are influenced not only by the aspect ratio but also by angles of inclination. The quantity of deflection in the mid-span and the force at the anchor point can be obtained by on-site measurement. The mechanical response study is very helpful for damage detection in health monitoring systems.

\section{Acknowledgement}

The work reported has been supported by the Science and Technology Project of Ministry of Housing and Urban-Rural Development (2010-K2-8), a project funded by the Priority Academic Program Development of Jiangsu Higher Education Institutions.

\section{References}

1. Basseville M., Bourquin F., Mevel L., Nasser H., Treyssede F., 2010, Handling the temperature effect in vibration monitoring: two subspace-based analytical approaches, Journal of Engineering Mechanics, 136, 3, 367-378

2. BouaAnani N., 2006, Numerical investigation of the modal sensitivity of suspended cables with localized damage, Journal of Sound and Vibration, 292, 3/5, 1015-1030

3. Cebalos M.A., Prato C.A., 2008, Determination of the axial force on stay cables accounting for their bending stiffness and rotational end restraints by free vibration tests, Journal of Sound and Vibration, 317, 1/2, 127-141 
4. Chen C.C., Wu W.H., Liu C.Y., 2012, Effects of temperature variation on cable forces of an extradosed bridge, 6th European Workshop on Structural Health Monitoring, Dresden, Germany, $1-8$

5. Cho S., Yim J., Shin S.W., Jung H.J., Yun C.B., Wang M.L., 2013, Comparative field study of cable tension measurement for a cable-stayed bridge, Journal of Bridge Engineering, 18, $8,748-757$

6. Deng Y., Ding Y., LI A., 2010, Structural condition assessment of long-span suspension bridges using long-term monitoring data, Earthquake Engineering and Engineering Vibration, 9, 1, 123-131

7. Hua X.G., Ni Y.Q., Chen Z.Q., Ko J.M., 2009, Structural damage detection of cable-stayed bridges using changes in cable forces and model updating, Journal of Structural Engineering, 135, 9, 1093-1106

8. Kangas S., Helmicki A., Hunt V., Sexton R., Swanson J., 2012, Cable-stayed bridges: case study for ambient vibration-based cable tension estimation, Journal of Bridge Engineering, 17, Special Issue: Nondestructive Evaluation and Testing for Bridge Inspection and Evaluation, 839-846

9. Kim B.H., PARK T., 2007, Estimation of cable tension force using the frequency-based system identification method, Journal of Sound and Vibration, 304, 3/5, 660-676

10. Kim B.H., Park T., Shin H., Yoon T.Y., 2007, A comparative study of the tension estimation methods for cable supported bridges, Steel Structures, 7, 77-84

11. KullaA J., 2003, Is temperature measurement essential in structural health monitoring, Proceeding of International Workshop on Structural Health Monitoring, Stanford University, Palo Alto, Calif., $717-724$

12. Lepidi M., Gattulli V., 2012, Static and Dynamic response of elastic suspended cables with thermal effects, International Journal of Solids and Structure, 49, 9, 1103-1116

13. Lepidi M., Gattulli V., Vestroni F., 2007, Static and dynamic response of elastic suspended cables with damage, International Journal of Solids and Structures, 44, 25/26, 8194-8212

14. Materazzi A.L., 2011, Eigenproperties of suspension bridges with damage, Journal of Sound and Vibration, 330, 26, 6420-6434

15. Mordini A., Savoy K., Wenzel H., 2008, Damage detection on stay cables using an open source-based framework for finite element model updating, Structural Health Monitoring, 7, 2, 91-102

16. Ni Y.Q., Ko J.M., Zheng G., 2002, Dynamic analysis of large-diameter sagged cables taking into account flexural rigidity, Journal of Sound and Vibration, 257, 2, 301-319

17. Peeters B., Maeck J., Deroeck G., 2001, Vibration-based damage detection in civil engineering: excitation sources and temperature effects, Smart Materials and Structures, 10, 3, 518-527

18. Ren W.X., Chen G., Hu W.H., 2005, Empirical formulas to estimate cable tension by cable fundamental frequency, Structural Engineering and Mechanics, 20, 3, 363-380

19. Treysssede F., 2009, Free linear vibrations of cables under thermal stress, Journal of Sound and Vibration, 327, 1/2, 1-8

20. TREYSSSEDE F., 2010, Vibration analysis of horizontal self-weighted beams and cables with bending stiffness subjected to thermal loads, Journal of Sound and Vibration, 329, 9, 1536-1552

21. Vanlanduit S., Parloo E., Cauberghe B., Guillaume P., Verboven P., 2005, A robust singular value decomposition for damage detection under changing operating conditions and structural uncertainties, Journal of Sound and Vibration, 284, 3/5,1033-1050

22. Xia Y., Hao H., Giovanna Z., Andrew D., 2006, Long term vibration monitoring of an RC slab: temperature and humidity effect, Engineering Structures, 28, 3, 441-452 
23. Zhou H., Ni Y., Ko J., 2010, Constructing input to neural networks for modeling temperaturecaused modal variability: mean temperatures, effective temperatures, and principal components of temperatures, Engineering Structures, 32, 6, 1747-1759

24. Zui H., Shinke T., Namita Y., 1996, Practical formulas for estimation of cable tension by vibration method, Journal of Structural Engineering, ASCE, 122, 6, 651-656

Manuscript received April 7, 2014; accepted for print June 3, 2014 